\title{
Interplay of Cavity Thickness and Metal Absorption in Thin-film InGaN Photonic Crystal Light-emitting Diodes
}

\section{Citation}

Rangel, Elizabeth, Elison Matioli, Hung-Tse Chen, Yong-Seok Choi, Claude Weisbuch, James S. Speck, and Evelyn L. Hu. 2010. Interplay of cavity thickness and metal absorption in thin-film InGaN photonic crystal light-emitting diodes. Applied Physics Letters 97, no. 6: 061118.

\section{Published Version}

10.1063/1.3480421

\section{Permanent link}

http://nrs.harvard.edu/urn-3:HUL.InstRepos:11130440

\section{Terms of Use}

This article was downloaded from Harvard University's DASH repository, and is made available under the terms and conditions applicable to Other Posted Material, as set forth at http:// nrs.harvard.edu/urn-3:HUL.InstRepos:dash.current.terms-of-use\#LAA

\section{Share Your Story}

The Harvard community has made this article openly available.

Please share how this access benefits you. Submit a story.

\section{Accessibility}




\title{
Interplay of cavity thickness and metal absorption in thin-film InGaN photonic crystal light-emitting diodes
}

\author{
Elizabeth Rangel, ${ }^{1, a)}$ Elison Matioli, ${ }_{1}^{1}$ Hung-Tse Chen, ${ }_{3}^{1}$ Yong-Seok Choi, ${ }^{1}$ \\ Claude Weisbuch, ${ }^{2}$ James S. Speck, ${ }^{1}$ and Evelyn L. $\mathrm{Hu}^{3}$ \\ ${ }^{1}$ Department of Materials, University of California, Santa Barbara, California 93106-5050, USA \\ ${ }^{2}$ Laboratoire de Physique de la Matière Condensée, Ecole Polytechnique, CNRS, 91128 Palaiseau, France \\ ${ }^{3}$ School of Engineering and Applied Physics, Harvard University, Cambridge, Massachusetts 02138, USA
}

(Received 9 June 2010; accepted 27 July 2010; published online 13 August 2010)

\begin{abstract}
Thin-film InGaN photonic crystal (PhC) light-emitting diodes (LEDs) with a total semiconductor thickness of either $800 \mathrm{~nm}$ or $3.45 \mu \mathrm{m}$ were fabricated and characterized. Increased directional radiance relative to Lambertian emission was observed for both cases. The 800-nm-thick PhC LEDs yielded only a slight improvement in total light output over the $3.45-\mu \mathrm{m}$-thick PhC LEDs. Simulations indicate that, except for ultrathin devices well below $800 \mathrm{~nm}$, the balance between $\mathrm{PhC}$ extraction and metal absorption at the backside mirror results in modal extraction efficiencies that are almost independent of device thickness, but highly dependent on mirror reflectivity. (C) 2010 American Institute of Physics. [doi:10.1063/1.3480421]
\end{abstract}

Photonic crystals $(\mathrm{PhCs})$ are now well recognized as a means to enhance the light extraction and emission directionality of InGaN light-emitting diodes (LEDs). However, several key design parameters for high-efficiency PhC LEDs remain to be fully characterized. One of the critical considerations for surface-patterned PhC LEDs is the thickness of the underlying semiconductor. In general, reduction of the semiconductor thickness will improve the overlap between the guided optical modes and the PhC layer. This is especially important for low-order modes, whose interaction with a shallow-etched PhC can be very weak. ${ }^{1}$ More specifically, simulations have shown ${ }^{2}$ that the extraction length of loworder modes-the $1 / e$ in-plane decay length as the modes are diffracted to air by the $\mathrm{PhC}-$ scales as $t^{3}$, where $t$ is the thickness of the unetched semiconductor below the $\mathrm{PhC}$, as shown in Fig. 1(a). Thus, holding all else constant, higher extraction efficiency is expected for thinner devices. In addition, it has been suggested that thinner PhC LEDs may afford more directional far-field patterns, since their emission is concentrated into a smaller number of guided modes. ${ }^{3,4}$

While the epitaxial thickness of conventional InGaN LEDs on sapphire is generally fixed by growth constraints to several microns or more, thin-film LEDs offer greater flexibility since the $n$-GaN can be thinned down after removal of the sapphire substrate. Several thin-film InGaN PhC LEDs with submicron thickness have now been reported, ${ }^{3-6}$ including a 700-nm-thick device with an unencapsulated extraction efficiency of $73 \% .^{5}$ However, the impact of thickness on the directionality and extraction efficiency has not been studied in detail. In particular, while the importance of metal absorption in these devices has been highlighted, ${ }^{3}$ the magnitude of this absorption loss as a function of cavity thickness has not been quantified.

In this letter, we report on an experimental comparison between thin $(800 \mathrm{~nm})$ and thick $(3.45 \mu \mathrm{m})$ vertically injected thin-film InGaN PhC LEDs. Despite a large difference in the number of guided modes supported by the two structures, the wavelength-integrated far-field patterns were re-

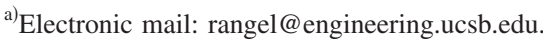

markably similar. In addition, the thin PhC LEDs exhibited only a small improvement in total output power over the thick PhC LEDs. This result is explained by simulated extraction and absorption lengths for low-order modes, which indicate that the balance between extraction and absorption is maintained as the structure is thinned to $800 \mathrm{~nm}$, resulting in an extraction efficiency that is largely independent of device thickness.

The epitaxial material was grown by metal-organic chemical vapor deposition on $c$-plane sapphire substrates. The structure consisted of an unintentionally doped GaN buffer, several microns of $n$-GaN, a 115 -nm-thick InGaN/ GaN 8× MQW active region emitting at $\lambda=450 \mathrm{~nm}$, a 15 $\mathrm{nm}$-thick AlGaN electron blocking layer, and a 265-nm-thick $p$-GaN layer. After deposition of a $\mathrm{Ni} / \mathrm{Ag}$ mirror as the $p$-contact, thin-film LEDs were fabricated by flip-chip bonding and laser lift off. The $n$-GaN was then thinned down by dry etching and mechanically polished to obtain a smooth surface. The remaining III-nitride thickness was $800 \mathrm{~nm}$ $(3.45 \mu \mathrm{m})$ for the thin (thick) structures. Hexagonal $\mathrm{PhCs}$ with an air fill factor of 30\% and lattice constant of $a$ $=448 \mathrm{~nm}$ were patterned by e-beam lithography and dry etched into the $n$-GaN to a depth of $240 \mathrm{~nm}$. Finally, an Al-based $n$-contact was deposited outside the PhC pattern. The side length of the LED mesa was $330 \mu \mathrm{m}$. An image of one of the PhC LEDs operating at $2 \mathrm{~mA}$ is shown in Fig. 1(b). The $\mathrm{PhC}$ is patterned in the four quadrants outside the cross-shaped $n$-contact, and recessed from the $n$-contact by $10 \mu \mathrm{m}$. The areas containing the $\mathrm{PhC}$ are visibly brighter

(a)
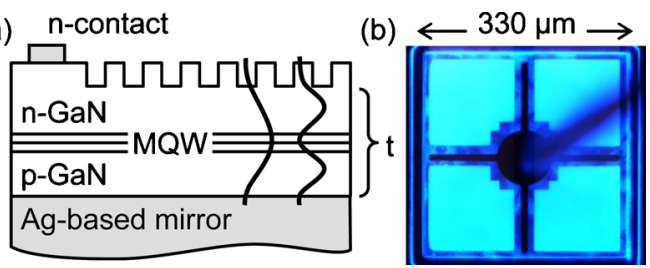

FIG. 1. (Color online) (a) Schematic cross section of the thin-film PhC LED structure, illustrating the $\mathrm{TE}_{0}$ and $\mathrm{TE}_{1}$ modes. (b) Top lit view of the thinfilm PhC LED. 


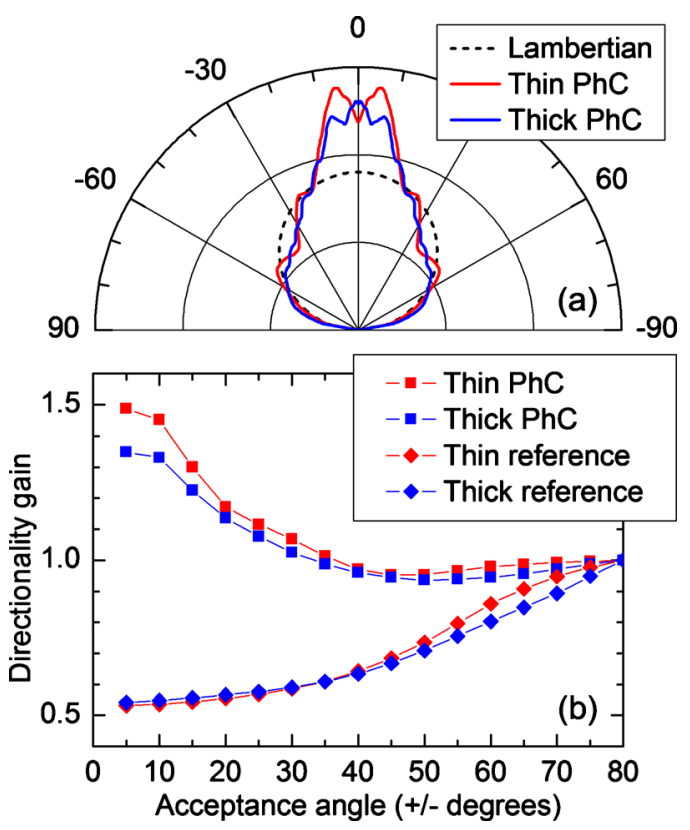

FIG. 2. (Color online) (a) Normalized far-field emission of 800-nm-thick and 3.45- $\mu \mathrm{m}$-thick PhC LEDs. (b) Directionality gain relative to Lambertian emission as a function of acceptance angle.

than the unpatterned regions between the $\mathrm{PhC}$ and $n$-contact.

Far-field patterns were measured by wavelength- and angle-resolved electroluminescence at $20 \mathrm{~mA}$. As expected, the large disparity in the number of guided modes supported by the thin and thick PhC LEDs-roughly eight TE modes in the thin LED compared to 34 in the thick LED-was reflected in the much richer PhC band structure measured for the thicker device (not shown). However, the overall shape of the wavelength-integrated far-field patterns was almost independent of the device thickness, as shown in Fig. 2(a). The data here has been averaged over a set of 120 measured in-plane angles in order to account for the azimuthal asymmetry of the PhC, and normalized such that the total integrated power is the same for both devices. The resultant patterns deviate substantially from one another only at small angles near the surface normal. At wider angles, where the intensity is determined by a larger number of guided modes and averaging effects prevail, the two curves are nearly identical.

The directionality of the far-field patterns can be quantified by comparison with a theoretical Lambertian emitter having the same total power as the PhC LEDs, as illustrated by the dashed curve in Fig. 2(a). The directionality gain is then defined as the power emitted into a given acceptance angle by the PhC LED, divided by the power emitted into the same aperture by the Lambertian emitter. ${ }^{4}$ As shown in Fig. 2(b), both the thin and thick PhC LEDs yielded a substantial directionality gain for small acceptance angles. This vertical directionality arises from the diffraction of highly excited, closely spaced low-order guided modes to the center of the air cone via second and third order diffraction at the chosen lattice constant. The similarity between the thin and thick PhC LEDs is a product of the fact that the guided modes in the thin structure, though more sparsely distributed, span nearly the same range in $k$-space as the guided modes in the thick structure. For comparison, the directionality gain of unpatterned reference LEDs on the thin and thick samples is also shown. Here the gain is less than unity due to the con-
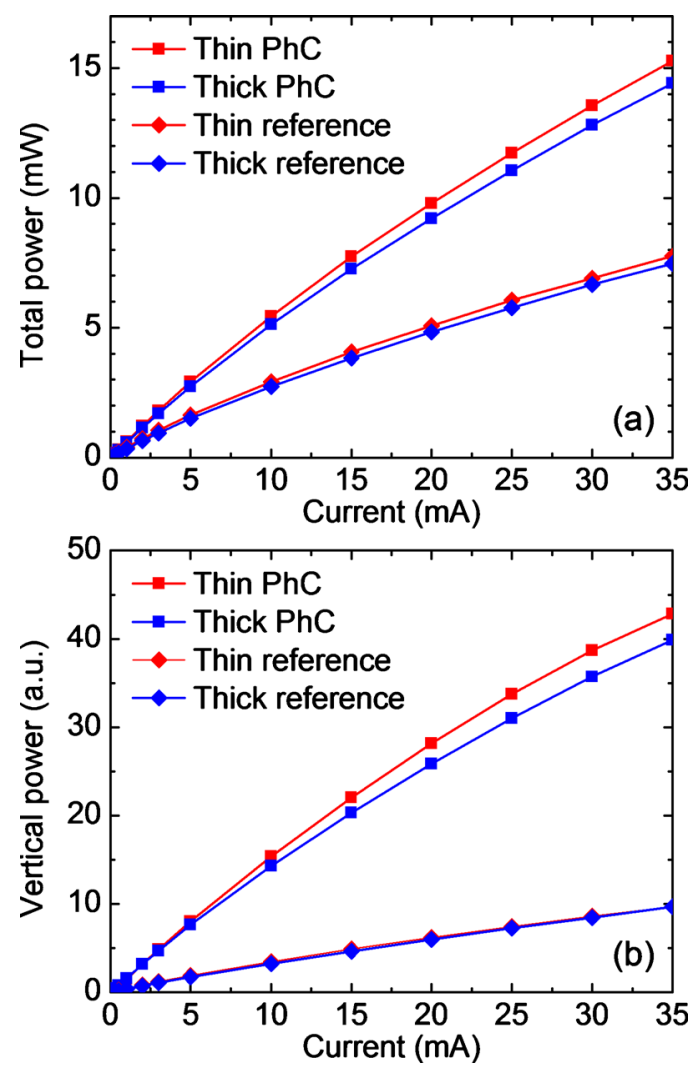

FIG. 3. (Color online) (a) Average total power vs current for the unencapsulated $\mathrm{PhC}$ and non-PhC LEDs. (b) Average power vs current collected with a limited acceptance angle of $\pm 20^{\circ}$. Same devices as in (a).

tribution of sidewall emission at large angles and the detuned half-cavity ${ }^{7}$ formed by the mirror and first quantum well. We note the excellent agreement between our results and those of Bergenek et al., ${ }^{4}$ who observed similar directionality gain for 850 -nm-thick PhC LEDs with an $a / \lambda$ ratio close to ours. It is also worth noting that the directionality gain will of course depend on what fraction of the total emission is due to $\mathrm{PhC}$ extraction.

The output power of the unencapsulated LEDs was measured under DC operation in an integrating sphere on TO-56 headers. The results are shown in Fig. 3(a), where the data have been averaged over all measured devices of each type. We note that no significant difference was observed between the unpatterned thin and thick LEDs. We attribute the absence of a microcavity enhancement ${ }^{8}$ (or suppression) of the extraction efficiency in the thin LEDs to a thickness variation in the order of $\pm 40 \mathrm{~nm}$ across the mesa, arising from the thinning and polishing process, which simulations indicate would be sufficient to average out any microcavity effects. ${ }^{9}$ More surprisingly, the efficiency of the PhC extraction also exhibited little dependence on the nominal device thickness. The average power of the thin PhC LEDs was just 6\% higher than that of the thick PhC LEDs, barely higher than the standard deviation of $4 \%$ within each set of devices.

The output power was also measured using a photodetector mounted above the sample with an acceptance angle of about $\pm 20^{\circ}$. As shown in Fig. 3(b), the thin PhC LEDs yielded an average enhancement over the unpatterned LEDs of 4.5 times-much higher than the enhancement in total power (1.9 times) — as a result of the vertical directionality of the $\mathrm{PhC}$ emission. However, the thickness of the devices still 

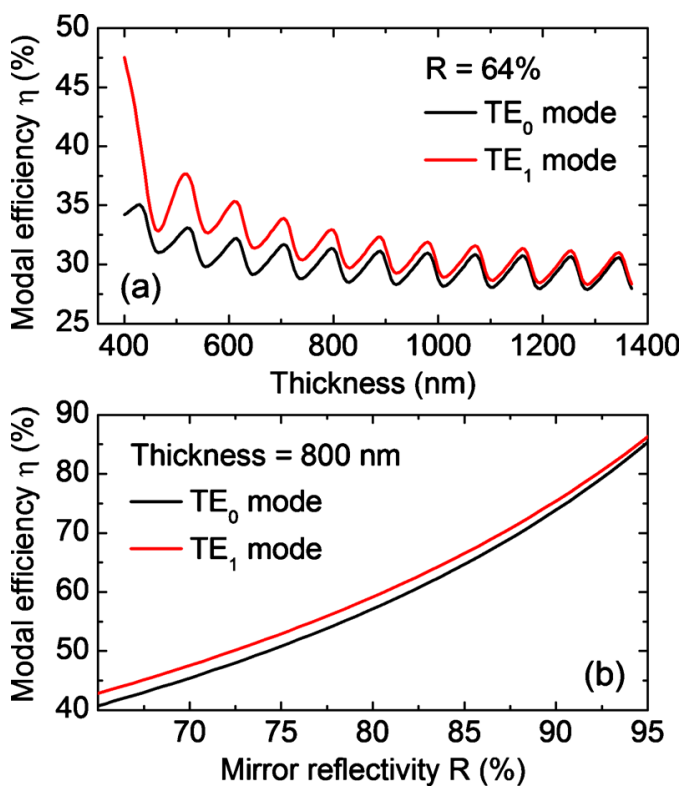

FIG. 4. (Color online) (a) Simulated modal extraction efficiency for the $\mathrm{TE}_{0}$ and $\mathrm{TE}_{1}$ modes as a function of the total PhC LED thickness. (b) Simulated modal extraction efficiency for the $\mathrm{TE}_{0}$ and $\mathrm{TE}_{1}$ modes in an 800-nm-thick $\mathrm{PhC}$ LED as a function of the mirror reflectivity.

had little impact; with the vertical power of the thin $\mathrm{PhC}$ LEDs just 9\% higher than that of the thick PhC LEDs.

As discussed above, the advantage of a very thin $\mathrm{PhC}$ LED is the improved overlap between low-order guided modes and the PhC layer. However, the thin nature of the structure also increases the overlap of these modes with the lossy metal mirror. To quantify the magnitude of these competing effects, we calculated the $1 / e$ decay lengths corresponding to absorption by the metal mirror and extraction to air by the $\mathrm{PhC}$-denoted by $L_{a b s}$ and $L_{e x}$ - using a threedimensional scattering matrix formalism. ${ }^{10}$ The simulated device had a hexagonal $\mathrm{PhC}$ with a lattice constant of $a$ $=200 \mathrm{~nm}$ and etch depth of $170 \mathrm{~nm}$. To approximate the unoptimized Ag-based mirrors used in this study, the complex refractive index of the mirror was taken to be $n=0.54$ $+2.47 i$, which yields a normal-incidence GaN-to-metal reflectivity of $R=64 \%$ at $\lambda=450 \mathrm{~nm}$.

Neglecting sidewall emission, the extraction efficiency of a single guided mode is given by

$$
\eta=\frac{1 / L_{e x t}}{1 / L_{e x t}+1 / L_{a b s}} .
$$

This modal efficiency is plotted in Fig. 4(a) for the $\mathrm{TE}_{0}$ and $\mathrm{TE}_{1}$ modes as a function of the device thickness. Raw simulations were convoluted with a thickness variation of \pm 40 $\mathrm{nm}$ to account for the nonuniformity in the real devices. Aside from periodic oscillations corresponding to vertical resonances with the $\mathrm{PhC}$, the modal extraction efficiency exhibits a weak downward trend as the thickness is increased from $400 \mathrm{~nm}$, with almost no dependence on thickness above $800 \mathrm{~nm}$. This is a result of the fact that any increase in $L_{e x t}$ is compensated by a corresponding increase in $L_{a b s}$. Notably, repeating the simulation for higher mirror reflectivities yielded the same trend, simply shifting the two curves upwards in efficiency.

To further explore the role of metal absorption in our thin PhC LEDs, the thickness of the simulated device was fixed at $800 \mathrm{~nm}$ and the normal-incidence mirror reflectivity in $\mathrm{GaN}$ was varied from $R=65 \%$ to $95 \%$ by varying the real component of the mirror's refractive index from $n=0.54$ to 0.01 , keeping the imaginary component fixed at $k=2.47$. As shown in Fig. 4(b), relatively small degradations in mirror reflectivity were found to correspond to large decreases in modal efficiency, due to large increases in modal absorption. For $R=89 \%$, corresponding to an ideal Ag mirror with a refractive index of $0.15+2.47 i,{ }^{11}$ the extraction efficiency of the $\mathrm{TE}_{0}$ mode is only $71 \%$. For $R=80 \%$, the extraction efficiency of the $\mathrm{TE}_{0}$ mode drops to $57 \%$. Given that low-order modes carry a substantial fraction of the total quantum well emission, these results highlight the critical importance of mirror quality in high extraction efficiency thin-film $\mathrm{PhC}$ LEDs.

In summary, we have fabricated and characterized thinfilm InGaN PhC LEDs with total semiconductor thickness of either $800 \mathrm{~nm}$ or $3.45 \mu \mathrm{m}$. Vertically directional light emission was observed in both cases. The output power of the $\mathrm{PhC}$ LEDs was found to be almost independent of the device thickness. These results are in good agreement with simulations, which indicate that the improvement in $\mathrm{PhC}$ extraction obtained by thinning the devices to $800 \mathrm{~nm}$ is largely negated by a corresponding increase in metal absorption. We conclude that except for extremely thin devices well below 800 $\mathrm{nm}$, the thickness of the PhC LED has little importance for either the directionality or the total extraction efficiency. In addition, we find that the extraction efficiency is strongly dependent on the optical properties of the metal mirror.

This work was supported by the Center for Energy Efficient Materials, an Energy Frontier Research Center funded by the U.S. DOE Office of Science, Office of Basic Energy Sciences under Award No. DE-SC0001009.

${ }^{1}$ A. David, C. Meier, R. Sharma, F. S. Diana, S. P. DenBaars, E. Hu, S. Nakamura, and C. Weisbuch, Appl. Phys. Lett. 87, 101107 (2005).

${ }^{2}$ A. David, H. Benisty, and C. Weisbuch, J. Disp. Technol. 3, 133 (2007).

${ }^{3}$ A. David, T. Fujii, B. Moran, S. Nakamura, S. P. DenBaars, and C. Weisbuch, Appl. Phys. Lett. 88, 133514 (2006).

${ }^{4}$ K. Bergenek, C. Wiesmann, H. Zull, C. Rumbolz, R. Wirth, N. Linder, K. Streubel, and T. F. Krauss, Proc. SPIE 7231, 72310C (2009).

${ }_{5}^{5}$ J. J. Wierer, A. David, and M. M. Megens, Nat. Photonics 3, 163 (2009).

${ }^{6}$ C.-F. Lai, J.-Y. Chi, H.-C. Kuo, H.-H. Yen, C.-E. Lee, C.-H. Chao, H.-T. Hsueh, and W.-Y. Yeh, Opt. Express 17, 8795 (2009).

${ }^{7}$ Y. C. Shen, J. J. Wierer, M. R. Krames, M. J. Ludowise, M. S. Misra, F. Ahmed, A. Y. Kim, G. O. Mueller, J. C. Bhat, S. A. Stockman, and P. S. Martin, Appl. Phys. Lett. 82, 2221 (2003).

${ }^{8}$ C. Weisbuch, A. David, T. Fujii, C. Schwach, S. P. DenBaars, S. Nakamura, M. Rattier, H. Benisty, R. Houdre, R. Stanley, J. F. Carlin, T. F. Krauss, and C. J. M. Smith, Proc. SPIE 5366, 1 (2004).

${ }^{9}$ Y.-S. Choi, M. Iza, E. Matioli, G. Koblmüller, J. S. Speck, C. Weisbuch, and E. L. Hu, Appl. Phys. Lett. 91, 061120 (2007).

${ }^{10}$ S. G. Tikhodeev, A. L. Yablonskii, E. A. Muljarov, N. A. Gippius, and T. Ishihara, Phys. Rev. B 66, 045102 (2002).

${ }^{11}$ E. D. Palik, Handbook of Optical Constants of Solids (Academic, San Diego, CA, 1998). 